\title{
Aneuploidy Presenting as Placental Thickness: A Case Report
}

\author{
Shefali Tyagi ${ }^{1}$, Swapnil Bhagat ${ }^{2}$
}

\begin{abstract}
A normally functioning placenta is critical for normal fetal growth and development. The size of placenta increases during fetal growth to allow it to do its vital functions. Thus, a normal development of placenta is important for an uneventful embryonic and fetal development. The case described here is a case of aneuploidy of chromosomes 13,16,21, and 22 diagnosed due to the detection of increased placental thickness.

Keywords: Aneuploidy, Thick placenta.

Journal of South Asian Federation of Obstetrics and Gynaecology (2021): 10.5005/jp-journals-10006-1947
\end{abstract}

\section{INTRODUCTION}

Normal fetal growth and development require the presence of a normally functioning placenta. The placenta increases in size during fetal growth and development to provide sufficient nutrition. Thus, the normal development of placenta is extremely important for proper development of the fetus.

The case described here is a case of aneuploidy of chromosomes $13,16,21$, and 22 diagnosed due to detection of increased placental thickness.

\section{Case Description}

A 33-year-old nonsmoking second gravida was presented in early pregnancy at 6 weeks. She had a daughter, 3.5 years old born by cesarean section due to nonprogress of labor. Her early pregnancy scan was normal. Nuchal translucency (NT) scan done at weeks was at low risk with NT being and other parameters for Down's within normal. Double marker done was at low risk. Her uterine artery resistance was high for which tablet aspirin $150 \mathrm{mg}$ was started. She was started on regular supplements of iron and calcium. At anomaly scan, the radiologist had a surprise. The three-fourth cavity of uterus was filled by the placenta and fetus was squeezed to one side. Anomaly scan revealed hyperechogenic bowel loops and small posterior fossa in fetus. There was a growth lag of 2 weeks for the fetus. The most noticeable finding was the hypertrophy of placenta (Fig. 1).

She was counseled for amniocentesis. Poor prognosis of pregnancy was explained to her. She did not agree to amniocentesis but wanted to terminate the pregnancy straight away and investigate the fetus postdelivery for abnormalities. As she was at 19 weeks, she could legally terminate the pregnancy. Her placenta was low lying as well. She was counseled about the risk of hemorrhage and need for hysterotomy or blood transfusion. With thorough counseling, she opted to try for termination by inducing labor. However, she started bleeding profusely when labor was induced by misoprostol and had to be taken for hysterotomy. A dead female fetus weighing $170 \mathrm{~g}$ was extracted. Placenta weighed around $500 \mathrm{~g}$ filling the entire uterine cavity. The procedure was uneventful. Postoperatively she did not consent for autopsy but karyotyping of fetus and placenta was sent. It showed tetrasomy of chromosomes 13, 16, 21, and 22.
${ }^{1}$ Department of Obstetrics and Gynecology, Cloudnine, Bengaluru, Karnataka, India

${ }^{2}$ Department of Radiology, Cloudnine, Bengaluru, Karnataka, India

Corresponding Author: Shefali Tyagi, Department of Obstetrics and Gynecology, Cloudnine, Bengaluru, Karnataka, India, Phone: +91 9945342657, e-mail: shefali.tyagi@hotmail.com

How to cite this article: Tyagi S, Bhagat S. Aneuploidy Presenting as Placental Thickness: A Case Report. J South Asian Feder Obst Gynae 2021;13(4):275-276.

Source of support: Nil

Conflict of interest: None

\section{Discussion}

The fetal growth and well-being are maintained by the placenta, which was described the first time in 1979 by Grannum et al. He talked about placental findings on ultrasound and its relation with fetal maturity in high-risk pregnancies. How well the fetus is being provided nutrition by the placenta can be assessed through sonography. It has been concluded in studies that placenta measures according to gestational age. If the placenta is thicker or thinner than expected, it might compromise fetal well-being. Adverse fetal outcomes were seen with thick placenta. It was realized that neonates with thick placenta fared poorly than with regular placenta.

Elchalal et al. ${ }^{1}$ concluded that if the placenta was thicker than $35 \mathrm{~mm}$ at 20-22 weeks or more than $51 \mathrm{~mm}$ at 32-34 weeks, it was considered to be a thick placenta. Placental thickness was contributed by its implementation site as well.

Lee et al. concluded that posteriorly implanted placenta was thicker than anterior placenta by 6-7 $\mathrm{mm}$. If posterior placenta was thicker than $40 \mathrm{~mm}$ or anterior was thicker than $33 \mathrm{~mm}$, it was considered out of range.

Many adverse outcomes, like abruption, increased admission to neonatal intensive care unit (NICU), congenital anomalies, fetal growth restriction, pregnancy-induced hypertension, preterm birth, poor Apgar score, low PH of umbilical artery, nonreassuring nonstress test, intrauterine fetal death, and gestational diabetes, were found to be associated more with thick placentas (Raio et al., ${ }^{2}$ Miwa et al., ${ }^{3}$ Elchalal et al., ${ }^{1}$ and Dombrowski et al.,' ${ }^{4}$ 1992).

(o) The Author(s). 2021 Open Access This article is distributed under the terms of the Creative Commons Attribution 4.0 International License (https://creativecommons. org/licenses/by-nc/4.0/), which permits unrestricted use, distribution, and non-commercial reproduction in any medium, provided you give appropriate credit to the original author(s) and the source, provide a link to the Creative Commons license, and indicate if changes were made. The Creative Commons Public Domain Dedication waiver (http://creativecommons.org/publicdomain/zero/1.0/) applies to the data made available in this article, unless otherwise stated. 


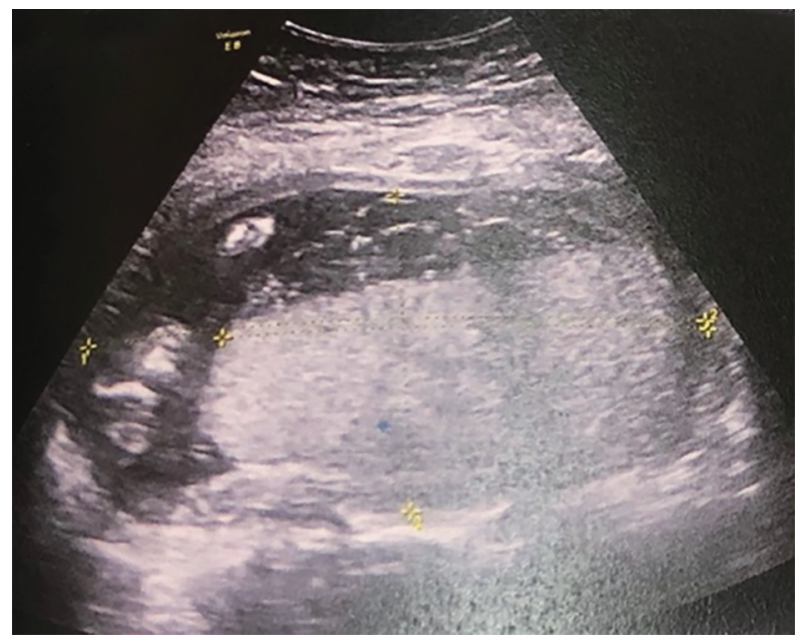

Fig. 1: Placenta at anomaly scan measured $14 \times 12 \times 5.6 \mathrm{~cm}$ filling three-fourth of uterine cavity

Sun et al. ${ }^{5}$ described that thick placenta can be a marker of aneuploidy. However, the literature search yields very few cases described citing an association between thick placenta and chromosomal aneuploidies.

Kashika ${ }^{6}$ concluded that placental thickness $>95$ th centile at 36 weeks compromised the fetal outcomes, with good Apgar scores in $20 \%$ of babies and poor Apgar scores in $80 \%$ of babies. All the babies were admitted to NICU.

Arabin et al. ${ }^{7}$ concluded that when the blood supply was studied between normal placenta and thick placenta, fetal and maternal blood waveforms were not much different between the two. It indicates that maybe ultrasonologically thick placenta represents a phase in placental malfunction.

Measuring placenta on ultrasound is easy and it is possible to include measurement in routine examination to detect high-risk pregnancy.

\section{CONCLUSION}

In conclusion, potentially serious maternal, fetal, and neonatal complications can be expected if placenta is thick and it seems that poor development of placental circulation leads to a compensation in form of greater thickness of placenta. The ultrasonographic measurement of placental thickness is a simple method to estimate placental size, and a thick placenta may be a useful predictor of adverse pregnancy outcomes. Therefore, placental thickness should be measured in addition to biometric parameters in antenatal women undergoing ultrasound.

A thick placenta should be regarded as a risk factor and needs good follow-up.

\section{Compliance with Ethical Standards}

Compliance, in accordance with the ethical standards, was maintained.

\section{References}

1. Elchalal U, Ezra Y, Levi Y, et al. Sonographically thick placenta: a marker for increased perinatal risk - a prospective cross-sectional study. Placenta 2000;21(2-3):268-272. DOI: 10.1053/plac.1999.0466.

2. Raio L, Ghezzi F, Cromi A, et al. The thick heterogeneous (jellylike) placenta: a strong predictor of adverse pregnancy outcome. Prenat Diagn 2004;24(3):182-188. DOI: 10.1002/pd.828.

3. Miwa I, Sase M, Torii M, et al. A thick placenta: a predictor of adverse pregnancy outcomes. SpringerPlus 2014;3:353. DOI: 10.1186/21931801-3-353.

4. Dombrowski MP, Wolfe HM, Saleh A, et al. The sonographically thick placenta: a predictor of increased perinatal morbidity and mortality. Ultrasound Obstet Gynecol 1992;2:252-255. DOI: 10.1046/j.14690705.1992.02040252.x

5. Sun X, Shen J, Wang L. Insights into the role of placenta thickness as a predictive marker of perinatal outcome. J Int Med Res 2021;49(2):030006052199096. DOI: 10.1177/0300060521990969.

6. Nagpal K, Mittal P, Grover SB. Role of ultrasonographic placental thickness in prediction of fetal outcome- a prospective Indian study. J Obstet Gynaecol India 2018;68(5):349-354. DOI: 10.1007/ s13224-017-1038-8.

7. Arabin $B$, Jimenez $E$, Vogel $M$, et al. Relationship of uteroand fetoplacental blood flow velocity wave forms with pathomorphological placental findings. Fetal Diagn Ther 1992;7(3-4): 173-179. DOI: 10.1159/000263695. 